\title{
Analyses of Time Division Multiple Access (TDMA) Schemes for Global Mobile Satellite Communications (GMSC)
}

\author{
D.S. Ilcev \\ Durban University of Technology, Durban, South Africa
}

\begin{abstract}
This paper provides analyzes of the Time Division Multiple Access (TDMA) techniques and their hybrids with Time Division Multiplexing (TDM) for implementation in Global Mobile Satellite Communications (GMSC). In satellite communication systems, and especially in GMSC networks, many users are active at the same time. The problem of simultaneous communications between many single or multipoint mobile satellite users, however, can be solved by using the Multiple Access Technique (MAT) schemes. Since the resources of the systems such as the transmitting power and the bandwidth are limited, it is advisable to use the channels with a complete charge and to create a different MAT to the channels. This generates a problem of summation and separation of signals in the transmission and reception parts, respectively. Deciding this problem consists in the development of orthogonal channels of transmission in order to divide signals from various users unambiguously on the reception part.
\end{abstract}

\section{INTRODUCTION}

Experimental TDMA systems were first developed since 1965 (Sekimoto and Puento, 1968). In fact, these early systems proved that time-interleaved, shortinterval communications were in fact technically feasible. Later systems (IEEE, 1979; Kwan, 1973; Maillet, 1972; Thompson, 1983; Watt, 1986) established that advanced operational TDMA concepts, such as high-accuracy, fast-acquisition synchronization, and high-capacity data formats were both possible and advantageous

In multiple access techniques for GMSC systems, the uplink carriers from Ground Earth Station (GES) terminals may be separate in time, not in frequency, code, or space. For instance, instead of assigning a frequency band, each ascending link is assigned a specific time interval and a given station transmits only during its assigned interval. This type of operation is it is called Time Division Multiple Access (TDMA) scheme. As can be seen, the TDMA scheme theoretically avoids the problem of many carriers trying to get through the satellite at the same time, thus avoiding the intermodulation problem of the Frequency Division Multiple Access (FDMA) scheme.

However, while the FDMA scheme involves relatively simple frequency tuning for accessing, and providing essentially independent channel on-off operation, the TDMA scheme requires communication concepts that are relatively new. To accommodate many users, TDMA time intervals must necessarily be short, requiring burst-type transmissions, and the time intervals of all users must be properly and accurately synchronized, requiring several levels of timing control. Thus, the required high-speed hardware for these operations is relatively new and, in many cases, is still under development. 


\section{PRINCIPLES OF TDMA SCHEME}

In the TDMA scheme, each uplink from GES is assigned a prescribed time interval in which to relay through the satellite. During its interval, a particular station has exclusive use of the satellite, and its uplink transmission alone is processed by the satellite for the downlink. Each carrier can use the same carrier frequency and use of the entire satellite bandwidth during its interval. Thus, since no other carrier uses the satellite during this time interval, no intermodulation or carrier suppression occurs, and the satellite amplifier can be operated in saturation so as to achieve maximal output power.

The TDMA downlinks always operate at full saturation power of the satellite, while the entire TDMA system must have all Earth terminals properly synchronized in time so that each can transmit through the satellite only during its prescribed interval, without interfering with the intervals of other stations. This time synchronization between satellite and all Earth stations is called network synchronization. A downlink Earth station terminals, wishing to receive the transmissions from a particular uplink, must gate into the satellite signal during the proper time interval. This means that all Earth stations, whether transmitting or receiving, must be part of the synchronized network. Many users of the TDMA satellite wish to establish a communication link in real-time, the total transmission time must be shared by all users. Thus, the time intervals of each Earth station must be relatively short and repeated at regular epochs. This type of short-burst, periodic operation is most conducive to digital operation, where each station transmits bursts of data bits during its intervals.

However, TDMA digital transmissions require that all receiving stations must obtain decoder synchronization in each interval, in addition to the required network synchronization for slot timing. For phase-coherent decoding, decoder synchronization requires establishing both a coherent phase reference and a coherent bit timing clock before any bits can be decoded within a slot. Also, word sync may be needed to separate the digital words occurring during a slot. This hierarchy of decoding synchronization must be established at the very beginning of each slot if the subsequent slot bits are to be decoded. Furthermore, since each slot contains data from a different source, synchronization must be separately established for each slot being received. In fact, even when receiving from the same station, synchronization must generally be reestablished from one periodic burst to the next. Hence, digital communications with TDMA has an inherent requirement for rapid synchronization in order to perform successfully. The technology for short-burst communications is rather new and will be closely linked to the development of high-speed digital processing hardware.

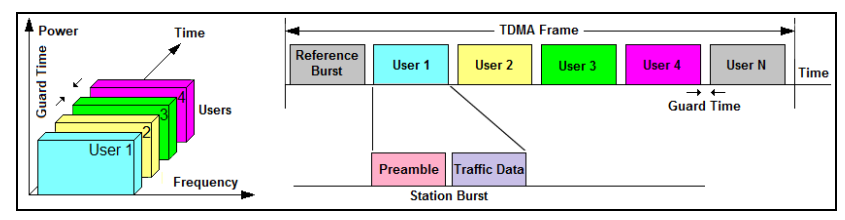

Figure 1. Time Division Multiple Access (TDMA) Techniques and TDMA Frame Structure

Therefore, the TDMA schemes permit more than two Mobile Earth Stations (MES) to use the same satellite network for interchanging information. Several transponders in the satellite payload share the frequency bands in use and each transponder will act independently of the others to filter out its own allocated frequency and further process that signal for transmission. This feature allows any GES located in the corresponding coverage area to receive carriers originating from several MES terminals and vice versa that carriers transmitted by one MES can be received by any GES terminal. This enables a transmitting GES to group several signals into a single, multi-destination carrier. Access to a transponder may be limited to a single carrier or many carriers may exist simultaneously. The baseband information to be transmitted is impressed on the carrier by the single process of multi-channel modulation.

\section{TIME DIVISION MULTIPLE ACCESS (TDMA) NETWORK CONCEPT}

The TDMA scheme is a digital access technique that permits individual satellite GES transmissions to be received by satellite in separate, non-overlapping time slots, called bursts, which contain buffered information. The satellite receives these bursts sequentially, without overlapping interference, and is then able to retransmit them to the MES terminal. Synchronization is necessary and is achieved using a reference station from which burst position and timing information can be used as a reference by all other stations. Each MES must determine the satellite system time and range so that the transmitted signal bursts, typically Quadrature Phase Shift Keying (QPSK) modulated, are timed to arrive at the satellite in the proper time slots. The offset QPSK modulation is used by Inmarsat-B MES. So as to ensure the timing of the bursts from multiple MES, TDMA systems use a frame structure arrangement to support telex (Tlx) in the mobile-to-shore direction. Therefore, a reference burst is transmitted periodically by a reference station to indicate the start of each frame to control the transmission timing of all data bursts. A second reference burst may also follow the first in order to provide a means of redundancy. In the proper manner, to improve the imperfect timing of TDMA bursts, several synchronization methods of random access, open-loop and closed-loop have been proposed.

In Figure 1 (Left) is shown a concept of TDMA, where each MES or user transmits a data burst with a guard time to avoid overlaps. Since only one TDMA burst occupies the full bandwidth of the satellite transponder at a time, input back off, which is needed to reduce Intermediate Frequency (IM) interference in 
FDMA, is not necessary for TDMA. At any instant in time, the transponder receives and amplifies only a single carrier. Thus, there can be no IM, which permits the satellite amplifier to be operated in full HPA saturation and the transmitter carrier power need not be controlled. Because all MES terminals to transmit and receive at the same frequency, tuning is simplified. This results in a significant increase in channel capacity. Another advantage over FDMA is its flexibility and time-slot assignments are easier to adjust than frequency channel assignments. The transmission rate of TDMA bursts is about $4,800 \mathrm{~b} / \mathrm{s}$, while the frame length is about 1.74 seconds and the optimal guard time is approximately $40 \mathrm{msec}$, using the open-loop burst synchronization method.

Accordingly, in the TDMA scheme, the transmission signals from various mobile users are amplified at different times but at the same nominal frequency, being spread by the modulation in a given bandwidth. Depending on the multiplexing techniques employed, two transmission hybrid schemes can be introduced for use in GMSC systems. The time slot in TDMA scheme is pre-assigned or can be changed on demand, and guard times are used between the time slots to avoid interference. Thus, the TDMA scheme is most practical for digital data transmission only, because of the burst nature of the transmissions. Downlink transmission consists of interleaved set of packets from all the ground stations. Two Reference Stations (RS), which could be one of the GES or a separate ground location, are used to establish the synchronization reference clock and provide burst time operational data to the network, and complex computer procedures, for automated synchronizations between MES terminals. The second disadvantage of TDMA scheme us that peak power and bandwidth of individual MES terminals need to be larger than with FDMA, owing to high burst bit rate.

However. the TDMA network offers a much more adaptive structure than FDMA regarding ease of reconfiguration for changing traffic demands. In Figure 1 (Right) is shown the signal structure of the TDMA network, consisting of $\mathrm{N}$ traffic stations or users slots. The total time period that includes all traffic station bursts and network information is called the TDMA frame. The frame repeats in time sequence and represents one complete transmission in the network. The frame times range from 1 to 20 ms and each station burst (slot) contains a preamble and traffic data (data bits). The preamble contains synchronization and station identification data. The reference burst, from the RS, is usually at the start of each frame and provides the network synchronization and operational information. Guard bands are included to prevent overlap and to account for different transmission times for each of the stations, based on their range to the satellite. Station bursts do not need to be identical in duration and can be longer for heavier traffic stations or during higher use periods. The specific allocation of burst times for each of the stations within the frame is called the burst time plan. The burst time plan is dynamic and can be changed as often as each frame to adapt to changing traffic patterns.

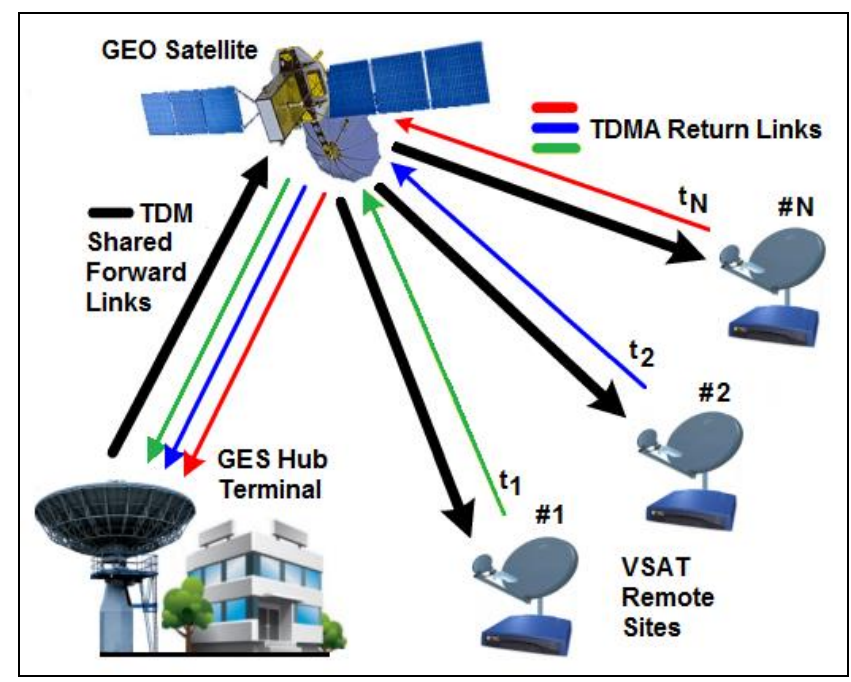

Figure 2. Hybrid TDM/TDMA Network Architecture

In general, preamble time should be long enough to establish reliable synchronization but should be short compared to the data transmission time. The ratio of preamble time to total slot time is sometimes called the preamble efficiency (॰p), or overhead. We often measure these times in numbers of bits or symbols and write this efficiency as follows:

$\eta_{P}=b_{T} / b_{F}=1-b_{O} / b_{F}$

or, in terms of the TDMA frame elements the efficiency will have the following relation:

$\eta_{P}=1-n_{R} \cdot b_{R}+n_{T} \cdot b_{T}+\frac{\left(n_{R}+n_{T}\right) \cdot b_{G}}{r_{T}} \cdot t_{F}$

where values $\mathrm{bT}=$ number of bits available for traffic, $\mathrm{bF}=$ total number of bits in frame, $\mathrm{bO}=$ number of overhead bits, $\mathrm{nR}=$ number of reference stations, $\mathrm{bR}=$ number of bits in reference burst, $\mathrm{nT}=$ number of traffic bursts, $b P=$ number of bits in traffic burst preamble, $\mathrm{bG}=$ number of bits in guard band, $\mathrm{rT}=$ total TDMA bit rate, in $\mathrm{b} / \mathrm{s}$, and $\mathrm{tF}=\mathrm{TDMA}$ frame time, in seconds (s).

The channel capacity for a TDMA network is most often evaluated in terms of an equivalent voicechannel capacity (nC). This allows evaluation of capacity for any type of data source bitstream: voice, data, video, or any combination of the three. The equivalent voice channel capacity is defined with the following relation:

$n_{C}=r_{I} / r_{C}$

where values $\mathrm{rI}=$ available information bit rate, and $\mathrm{rC}=$ equivalent voice channel bit rate. 
4 HYBRID TDM/TDMA NETWORK ARCHITECTURE

The Time Division Multiplexing (TDM) scheme is a method of transmitting and receiving independent signals over a common signal path by means of synchronized switches at each end of the transmission line so that each signal appears on the line only a fraction of time in an alternating pattern. It is a communication process that transmits 2 or more digital signals or analog signals over a common channel. The TDM program is used for long-distance communication links and bears heavy data traffic loads from end-users, so it can be further extended into the TDMA scheme, where several stations connected to the same physical medium, for example sharing the same frequncy channel, can communicate.

Satellite links normally relay many signals from many MES but to avoid interfering with each other it is necessary for some kind of separation or division. This separation is known as multiplexing and its common forms are Frequency Division Multiplexing (FDM) and TDM. The TDM is easier to implement with digital modulation and to form hybrid solutions applicable to all types of baseband signals.

The first generation of the Inmarsat analog standard-A MES uses the TDM/TDMA arrangement for telex transmission, which scenario is shown in Figure 2. Each MES has at least one TDM carrier and each of the carriers has 20 telex channels of 50 bauds and a signaling channel. Moreover, there is also a common TDM carrier continuously transmitted on the selected idle listening frequency by the Network Coordination Station (NCS) for out-of-band signaling. The MES remains tuned to the common TDM carrier to receive signaling messages when the mobile is idle or engaged in a telephone call. When an MES is involved in a telex forward call it is tuned to the TDM/TDMA frequency pair associated with the corresponding GES to send messages in shore-tomobile direction. Telex transmissions in the return mobile-to-shore direction form a TDMA assembly at the satellite transponder. Each frame of the return TDMA telex carrier has 22 time slots, while each of these slots is paired with a slot on the TDM carrier. The allocation of a pair of time slots to complete the link is received by the MES on receipt of a request for a telex call. Otherwise, the Inmarsat-A uses for forward signaling a telex mode, while all other Moile Satellite System (MSS) Inmarsat standards for forwarding signaling and assignment channels use the TDM Binary Phase Shift Keying (BPSK) scheme.

The new generation of Inmarsat digital standard-B (inheritor of standard-A) uses the same modulation TDM/TDMA technique but instead of Aloha BPSK $(\mathrm{BCH})$ at a data rate of $4800 \mathrm{~b} / \mathrm{s}$ for the return request channel used by Inmarsat-A, new standard-B is using Aloha Offset. Quadrature Phase Shift Keying (OQPSK), 1/2 - FEC, at a data rate of $24 \mathrm{~Kb} / \mathrm{s}$. This MAT satellite network is also useful for the Inmarsat standard-C MES terminal for maritime, land (road and rail), and aeronautical applications. In this case, the forward signaling link and sending of messages in the ground-to-mobile direction use a fixed assigned TDM carrier. The return signaling channel uses hybrid, slotted Aloha BPSK (1/2 FEC) with a provision for receiving some capacity and the return message channels in the mobile-to-ground direction are modulated by the TDMA system at a data rate of $600 \mathrm{~b} / \mathrm{s}$.

The TDM/TDMA technology uses a single highspeed TDM carrier transmitted from the central GES site or Hub, from which many Very Small Aperture Terminal (VSAT) stations can receive information. For this TDM forward link, the DVB-S2 of Digital Video Broadcasting-Return Channel via Satellite (DVB-RCS) standard is most commonly used. It is also the most flexible for multiplexing many concurrent streams of traffic to different sites, and the most efficient with its support of Adaptive Coding and Modulation (ACM). The ACM mode dynamically adjusts the modulation and coding on the "virtual link" to each VSAT individually, as local conditions (e.g., weather, interference) at the VSAT change. To transmit back to the central site efficiently, the VSATs in a TDM/TDMA network are synchronized, and they transmit information in "burst mode" within a series of short, scheduled timeslots. Timeslots may be assigned across multiple TDMA carriers and accessed using "fast frequency hopping". Timeslots are assigned to each VSAT exclusively (i.e., without contention) based on their current traffic needs. This is called Dynamic TDMA, and it is the most advanced form of TDM/TDMA.

The TDMA technology is fully standardized internationally by the DVB group under the DVBRCS family of standards. In Table 1 are presented the advantages and disadvantages characteristics of the TDM/TDMA technology with the cost of remote (VSAT). For instance, the low-cost TDMA/DVB-RCS Indoor Unit (IDU) or VSAT stations have dropped in price to $\$ 1,000$ including Outdoor Unit (ODU) or VSAT antenna, while the cost of SCPC modems is $\$ 6,000$. The Antenna unit and its ODU sizing are based on either shared carrier size or dedicated carrier size. Thus, the supplying cost advantages of TDM/TDMA presented earlier still apply when comparing against SCPC with bandwidth cancellation.

Table 1. List of TDM/TDMA Characteristics

\begin{tabular}{|l|l|}
\hline \multicolumn{1}{|c|}{ Advantage } & \multicolumn{1}{c|}{ Disadvantage } \\
\hline Sharing of satellite bandwidth & $\begin{array}{l}\text { High Latency and Increased } \\
\text { Jitter }\end{array}$ \\
\hline $\begin{array}{l}\text { Lower overall OPEX compared } \\
\text { to dedicated pipes }\end{array}$ & $\begin{array}{l}\text { Demanding remotes can burden } \\
\text { the system }\end{array}$ \\
\hline $\begin{array}{l}\text { Good for low data rate } \\
\text { applications }\end{array}$ & $\begin{array}{l}\text { Fragmentation of packets. Less } \\
\text { effective for voice and video }\end{array}$ \\
\hline Low cost remotes & Expensive hub equipment \\
\hline Large population of users & $\begin{array}{l}\text { All remotes must be designed } \\
\text { around worst case link }\end{array}$ \\
\hline
\end{tabular}

The TDMA networks allow all VSATs to dynamically share multiple TDMA carriers as if they were a single large pool of bandwidth. Each TDMA carrier group may contain dozens of carriers, with up to 32 carriers per carrier group in a satellite network. Therefore the "return link" may contain huge amounts of capacity, in aggregate. In the TDM/TDMA 
network, the TDMA carriers may operate at widely different symbol rates, e.g., from $500 \mathrm{ks} / \mathrm{s}$ to $5 \mathrm{Ms} / \mathrm{s}$ and even higher. To determine which VSAT will use which timeslots on which carrier at any moment, the satellite link uses Adaptive Carrier Selection (ACS). The ACS mode is applied dynamically for each VSAT, given its local weather conditions, configuration, e.g., antenna and Block Upconverter (BUC) size, and service policy, e.g., maximum rate requirements. In fact, the ACS mode determines what carrier \& symbol rate will work best at the current signal levels of those available in the carrier group.

In addition, in a satellite DVB-RCS2 (2nd Generation) network, ACM per burst is supported for each VSAT and on all TDMA carriers in the carrier group. This further optimizes efficiency, throughput, and reliability for each VSAT and greatly simplifies network operations. Any VSAT can use any MODulation and CODing (MODCOD), on any carrier, if necessary. DVB-RCS2 SatLink TDM/TDMA networks now surpass SCPC networks not only in efficiency, but also in throughput and link availability for almost any conceivable network configuration and satellite bands, such as $\mathrm{C}, \mathrm{Ku}$, and Ka-band domain.

\section{HYBRID TDM/SCPC NETWORK ARCHITECTURE}

The TDM/TDMA and Single Channel Per Carrier (SCPC) architectures are the main alternative technologies for satellite networking in the world today. The modem and management technologies underlying both approaches have been advancing rapidly in recent years, causing some confusion as to which technology is better for a given set of networking requirements. The SCPC network refers to using a single signal at a given frequency and bandwidth. Most often, this is used on broadcast satellites to indicate that radio stations are not multiplexed as subcarriers onto a single video carrier, but instead independently share a transponder.

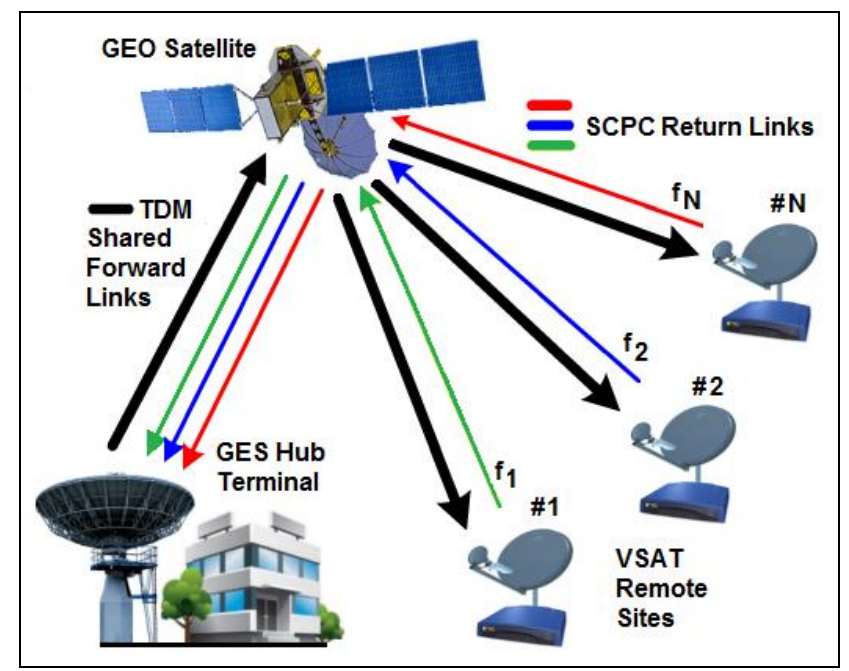

Figure 3. Hybrid TDM/SCPC Network Architecture

The SCPC mode is using for a VSAT satellite transmission system that uses a separate frequency carries for each of its communication channels, as opposed to frequency division multiplexing that combines many channels on a single carrier. It can be used for broadcast data and full-duplex audio and video communications. In an SCPC system, transmissions are sent to the satellite continuously on a single satellite carrier. The satellite signal is received at a single location, in the case of a point-to-point system, or at many different locations in a broadcast system, providing hubless connectivity among multiple sites. This technology a system where each sub-division carries only one $4-\mathrm{kHz}$ voice channel enables companies and corporate organizations to establish their own private network to connect sites into a single network with highly reliable performance with very low latency.

Due to the increasing dominance of IP traffic, many former SCPC networks have already been converted to TDM/TDMA network architecture. However, some SCPC networks have converted only "half-way", whereby a DVB-S2 TDM carrier is used on the forward link, but SCPC links are used for return link communications. This hybrid configuration is called TDM/SCPC scheme and its network architecture is illustrated in Figure 3. If using DVB-S2 it gets the full benefits of statistical multiplexing and ACM on the forward link, but these benefits are non-existent on the return link in this hybrid network. Therefore, the technical and business rationales for using the TDM/SCPC hybrid networks are weak at best.

Nonetheless, the TDM/SCPC hybrid configuration is commonly promoted and used in certain types of VSAT networks, in particular in cellular backhaul networks and in some other types of networks where fast access to large amounts of capacity for the return link (upstream) traffic must be guaranteed. There are four possible reasons for the continued use of this form of SCPC confirguration:

1 The possibility that SCPC in "continuous mode" will provide better modem efficiency (in $\mathrm{b} / \mathrm{s}$ per $\mathrm{Hz}$ ) than TDMA burst mode due to lower overhead and ability to use higher-rate, more efficient MODCOD scheme;

2 The possibility that SCPC links are better at providing guaranteed capacity and will operate more reliably against rain fades, interference, or congestion;

3 The possibility that SCPC links will provide lower latency or less total delay; and

4 The pssibility that SCPC links can be operated at a higher speed, when necessary, for any or all sites within the satellite transponder footprint.

These possibilities or some of them are true with respect to the limitations of some popular TDM/TDMA technologies. For those technologies, the hybrid TDM/SCPC option is useful and may even be "cost-effective" in networks with nearly constant levels of traffic in the peak hour at each site, a consistent peak hour time each day. In Table 2 are presented the advantages and disadvantages characteristics of the TDM/SCPC technology with the cost of remote (VSAT). 
Table 2. List of TDM/SCPC Characteristics

\begin{tabular}{|l|l|}
\hline \multicolumn{1}{|c|}{ Advantage } & \multicolumn{1}{|c|}{ Disadvantage } \\
\hline $\begin{array}{l}\text { Dedicated bandwidth for each } \\
\text { remote inbound }\end{array}$ & $\begin{array}{l}\text { Each remote requires its own } \\
\text { space segment }\end{array}$ \\
\hline $\begin{array}{l}\text { Provides superior Quality of } \\
\text { Service for mission critical } \\
\text { applications }\end{array}$ & $\begin{array}{l}\text { Expensive OPEX if each remote } \\
\text { bandwidth is not fully utilized }\end{array}$ \\
\hline Low Latency and Low Jitter & $\begin{array}{l}\text { SCPC modems typically more } \\
\text { expensive than TDMA modems }\end{array}$ \\
\hline $\begin{array}{l}\text { Best transmission method for } \\
\text { real-time applications, voice, } \\
\text { data, video, broadcast, etc. }\end{array}$ & $\begin{array}{l}\text { Fixed data rates on the inbound } \\
\text { links }\end{array}$ \\
\hline
\end{tabular}

However, in comparison to satellite TDM/TDMA networks using the DVB-RCS2 standards, these conditions do not hold true. In fact, the opposite is true, because, in terms of total network efficiency, a satellite DVB-RCS2 return link operating in TDMA burst mode will deliver $2 \mathrm{x}$ more in bps/Hz than some popular SCPC options, even before adding in the benefits of statistical multiplexing with TDMA configuration.

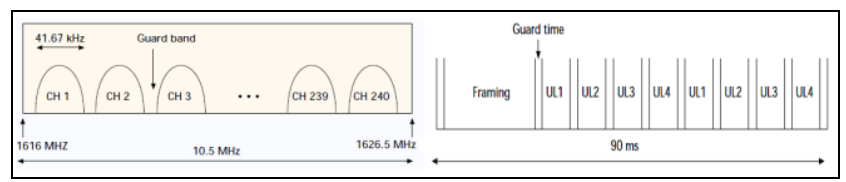

Figure 4. Iridium FDMA Scheme and TDMA Frame Structure

\section{HYBRID FDMA/TDMA NETWORK ARCHITECTURE}

The Iridium GMSC system employs a hybrid FDMA/TDMA access scheme, which is achieved by dividing the available $10.5 \mathrm{MHz}$ bandwidth into 150 channels introduced into the FDMA components. Each channel accommodates a TDMA frame comprising eight-time slots, four for transmission, and four for the sgnal reception. Each slot lasts about $11.25 \mathrm{msec}$, during which time data are transmitted in a $50 \mathrm{~Kb} / \mathrm{s}$ burst. Thus, each frame lasts $90 \mathrm{msec}$ and a satellite is able to support 840 channels. In such a way, a mobile satellite user is allocated a channel occupied for a short period of time, during which transmissions occur. The Iridium satellite system supports full-duplex voice channels at $4800 \mathrm{~b} / \mathrm{s}$ (2400 $\mathrm{b} / \mathrm{s}$ according to and half-duplex data channels at $2400 \mathrm{~b} / \mathrm{s}$. The IRIDIUM network utilizes multiple spot beams on each satellite that divide the satellite footprint into smaller cells. However, to provide twoway satellite communications, the IRIDIUM system uses a combination of Frequency Division Multiple Access (FDMA) and Time Division Multiple Access (TDMA) techniques.

The Hybrid FDMA/TDMA Network Architecture is established when two slots (same position in time) of the user are allocated in two different narrow-band radio channels. Iridium satellite system uses frequencies in the L-band of $1616 \mathrm{MHz}$ to $1626.5 \mathrm{MHz}$ for the user's uplink and downlink with the satellites. This gives the system $10.5 \mathrm{MHz}$ of bandwidth. As shown in Figure 4 (Left), the Iridium FDMA scheme divides the available bandwidth into 240 channels of $41.67 \mathrm{kHz}$ for a total of $10 \mathrm{MHz}$. This leaves $500 \mathrm{kHz}$ of bandwidth for guard bands, which amounts to approximately $2 \mathrm{kHz}$ of guard band between channels.

The TDMA frame is $90 \mathrm{~ms}$ long and it contains four full-duplex user satellite channels at a burst data rate of $50 \mathrm{~kb} / \mathrm{s}$. The four full-duplex channels consist of four uplink time slots and four downlink time slots, as depicted in Figure 4 (Right). The eight user time slots take up a total of $69.12 \mathrm{~ms}$, which leaves $20.88 \mathrm{~ms}$ of the TDMA frame for framing bits and guard time slots. A possible frame structure is to use a framing time slot twice as long as an individual user time slot. This would result in 864 framing bits taking up $17.28 \mathrm{~ms}$. Subtracting this value from the $20.88 \mathrm{~ms}$ remaining in the TDMA frame leaves $3.6 \mathrm{~ms}$ for guard time slots. This can be divided into eight 400 microsecond guard time slots between time slots in the frame, and two 200 microsecond guard time slots at each end of the frame. Although the exact frame structure is not published in the open literature, this approach is reasonable. Thus, it uses 4.6 percent of the $90 \mathrm{~ms}$ frame for guard timeand utilizes 76.8 percent of the frame for actual data bits.

\section{CONCLUSION}

The performances and capacities of the Time Division Multiple Access (TDMA) network architectures and their hybrids with Time Division Multiplexing (TDM)for GMSC applications have been analyzed an implemented many years ago for an $\mathrm{C}, \mathrm{Ku}$ and newest Ka-band. The Multiple Access Technique (MAT) schemes are is the use of multiplexing techniques to provide communication service to multiple fixed and mobile satellite users over a single channel. It allows for many users at one time by sharing a finite amount of spectrum. The TDM/TDMA, Single Channel Per Carrier (SCPC), and their hybrid solutions are the main alternative technologies for mobile satellite network architectures in the world today. The VSAT satellite modems and management technologies underlying both approaches have been advancing rapidly in recent years, causing some confusion as to which technology is better for a given set of networking requirements. In fact, the main architecture for design hybrid TDMA satellite networks is Time Division Multiplexing (TDM) technique and its combination with TDM/TDMA, TDM/SCPC and FDMA/TDMA hybrid network architecture for GMSC applications. In such a way, implementing these hybrid MAT networks promise many improrovements in satellite transmission systems.

The Frequency Division Multiple Access (FDMA) technique is widely used in the general analog telecommunication and satellite communications systems for all mobile applications at sea, on the ground, and in the air. The working principle of the FDMA as usual is dividing the signaling dimensions along the frequency axes to create many separate channels. After that, allocates these channels to fixed or mobile satellite users. The guard bands have an 
important effect on decreasing the transmission impairments. The FDMA technique has many advantages such as good capacity, simple algorithms, and so on. Despite the advantages, FDMA has many disadvantages such as constant data rate and channel capacity. Therefore, the FDMA technique is used in many applications such as analog cellular and satellite systems.

On the other hand, the TDMA technique is an advanced digital multiple access technology that allows more than one user to access radio frequency (RF) channels in satellite and other telecommunications systems. The principle work of TDMA is that the signals should be divided into milliseconds-long packets. Then, allocating a single frequency channel for short time and then moving to another channel to give it its own interval. Like the FDMA technique, the TDMA scheme has guard times that prevent any interference between channels, and decrease the factors of transmission impairments. The TDMA technique has many advantages such as good capacity with high data rates. However, the TDMA scheme has many disadvantages such as attenuation impairment.

\section{REFERENCES}

[1] Richharia M. (2001), “Mobile Satellite Communications - Principles and Trends", Addison-Wesley, Harlow, UK, 560.

[2] ETRI, (2005), “Mobile DVB-RCS Systems", Daejeon, South Korea, 23.

[3] Ilcev, (2017), “Global Mobile Satellite Communications for Maritime, Land and Aeronautical Applications Volume2", Springer, Boston, US, 686.

[4] Maral G. (2003), "VSAT Networks", John Wiley, Chichester, UK, 296.

[5] Ilcev D. S. (2011), "Global Mobile CNS", Manual, CNS Systems, Durban, South Africa, 285.

[6] Maini A.K. \& Agrawal V., (2007), "Satellite Technology - Principles and Applications", John Wiley, Chichester, 696.

[7] Kolawole M.O., (2002), "Satellite Communication Engineering", Marcel Dekker, New York, 269.

[8] Pratt S.R. at all, (1999), "Operational and Performance Overview of the Iridium Low Earth Orbit Satellite System", IEEE Communications Surveys, New York, 9.

[9] STM, (2013), TDMA vs. SCPC STM Group, Inc. Lysaker, Norway, 13.

[10] Gigard R, (2020), "Satellite Network Design", Intelsat, McLean, VA, 53.

[11] Gagliardi R.M., (1991), "Satellite Communications", Van Nostrand Reinhold, New York, 592.

[12] Ohmory S., Wakana. H \& Kawase S. (1998), “Mobile Satellite Communications", Artech House, Boston, 466.

[13] EMC, (2015), "TDMA vs. SCPC SatLink Technical Note", EMC Satcom Technologies, Lysaker, Norway, 13.

[14] Garvey G., (2013), "Multiple Access Techniques", Atlanta RF, LLC, Roswell, GA, 53.

[15] Sheriff R.E. \& Hu Y.F., (2001), "Mobile satellite communication networks", John Wiley, Chichester, 381. 